\title{
Research on the structure of energy consumption pattern in typical Western China based on time series analysis
}

\author{
Weizheng Kong ${ }^{1, a}$, Hongcai Dai ${ }^{1}$, Yaohua Wang ${ }^{1}$, Xiaoyu $\mathrm{Wu}^{1}$ and Rui Chen ${ }^{1}$ \\ ${ }^{1}$ State Grid Energy Research Institute Co. Ltd, Binhe Road 18\#, Future Science Park, Changping, Beijing 102209, China
}

\begin{abstract}
Accelerating the transformation of the energy consumption pattern in western China and promoting the development of clean energy are the main problems facing the energy consumption system. This paper bases on the characteristics of China's western region the industrial structure, the energy consumption structure, and analyses the energy consumption model transformation trend of typical of the west, on this basis, the combination of time series analysis and ARIMA model is used to set up different typical energy consumption in the field of forecast and analysis, and put forward according to the results of the analysis of energy consumption model transformation.
\end{abstract}

\section{Introduction}

Along with the global trend of low carbon, China's economy and society are also stepping into a new journey. China's economy will gradually shift from "structural growth" to "structural deceleration". In this critical period of development and transformation, we should get rid of the inherent mode of growth mechanism and find new internal driving force for growth and leaping. The key to development is to break through the constraints of resources and other aspects, and form high-quality resources and environment matching the stage of economic development. The western region of China has a very rich resource reserve. Accelerating the transformation of the energy consumption pattern and promoting the development of clean energy is one of the main problems that the energy consumption system must face. Along with the reform of the world's energy structure, the current energy supply and demand change one of the most obvious sign, is gradually to clean energy to replace fossil energy, the formation of coal, oil, gas, nuclear and new energy, renewable energy and other diversified energy supply, to low-carbon clean energy as the forerunner of comprehensive energy era is coming. The western region is one of the regions with the most abundant clean energy resources in China, and has a very rich energy reserve, which provides a good foundation for the construction of a comprehensive energy system. While protecting the environment, we will strive to improve the energy consumption structure, optimize the energy supply system, and accelerate the reform of the energy system. Based on the characteristics of energy structure in western China, this paper analyzes the influencing factors of energy consumption pattern and discusses the changing trend of energy consumption pattern.
At present, scholars at home and abroad study the energy structure from their own perspectives. Ref.[1] points out that the energy consumption in the BeijingTianjin-Hebei region is dominated by coal and oil, and the proportion of renewable energy in the energy consumption structure is small, lower than the national average level. In [2],Xu analyzed the energy consumption structure in China's rural areas and pointed out that with the development of China's rural economy and the increase of residents' income, the proportion of various energy consumption has changed significantly, rural residents' energy consumption tends to be commercialized and the proportion of renewable energy consumption is on the rise. The literature [3], the author, on the basis of current situation of the development of cities in our country, using the method of system dynamics, builds the system dynamics model of urban energy consumption, the energy consumption of the whole system is divided into four subsystems, the dynamic simulation results show that energy consumption, reasonable control three industrial structure can reduce energy consumption. Literature [4] points out that increasing energy prices can increase the consumption proportion of new energy and clean energy, and identifies the influence channels of energy prices based on the VAR model. Literature [5-7] introduces the influence of financial subsidies, policies and technologies on energy consumption structure.The current analysis of energy consumption structure are mainly concentrated in the use of long-term history data for artificial analysis of energy consumption structure, and analysis the point of view of focus on the influence factors of energy consumption structure, forecasting, and the effect of energy consumption structure adjustment, but the light of the regional energy consumption structure of a lack of effective analysis means. Therefore, this paper adopts the time series analysis method to

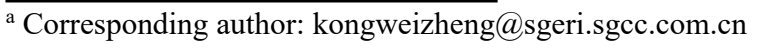


establish the autoregressive summation ARIMA model to analyze the trend characteristics of energy consumption and the derivative industry model in the future.

\section{Study on energy consumption pattern structure based on time series analysis method}

In the 1970s, American scholar Box and British statistician Jenkins put forward a set of methods about time series analysis, prediction and control, which is called Box-Jenkins method. In general, the modelling of time series can be divided into three stages: initial model identification, determining the model type that time series should belong to. The basic principle is to preliminarily judge the model type suitable for time series according to the truncation and trailing of data autocorrelation and partial correlation functions. The parameters of the model were estimated by the order criteria. The applicability test of the model. Box-Jenkins model requires time series to be stationary series, while the time series of things or systems in natural phenomena and economic phenomena are often nonstationary series, so summing autoregressive moving average model (ARIMA) is needed for modelling.

\subsection{The basic idea of ARIMA model}

The basic idea of the ARIMA model is as follows: for the non-stationary time series, it is made into a stationary series by using some order difference; The possible $\mathrm{P}$ and $\mathrm{Q}$ parameters in the model were preliminarily proposed by using autocorrelation and partial autocorrelation function graphs. Then, parameter estimation and model diagnosis were used to determine the $\mathrm{P}$ and $\mathrm{Q}$ parameters in the model by $\mathrm{AIC}$ and BIC. The smaller the values of AIC and BIC, the better the model fitting was. The residual sequence of the fitting model is tested. If the residual sequence is listed as a white noise sequence, it means that the information in the original sequence has been fully extracted through model fitting, and the established model is appropriate. Otherwise, the above steps need to be repeated to rebuild the model until the residual sequence is listed as a white noise sequence. Finally, forecast, use the fitting optimal model to make a back substitute prediction of energy consumption, and forecast the development trend. In the $\operatorname{ARIMA}(p, q, d)$ model, AR is autoregressive, $P$ is the number of autoregressive terms, MA is the moving average, $\mathrm{Q}$ is the number of moving average terms, and $\mathrm{D}$ is the number of difference times when the time series is stabilized. The specific steps are shown in Figure.1.

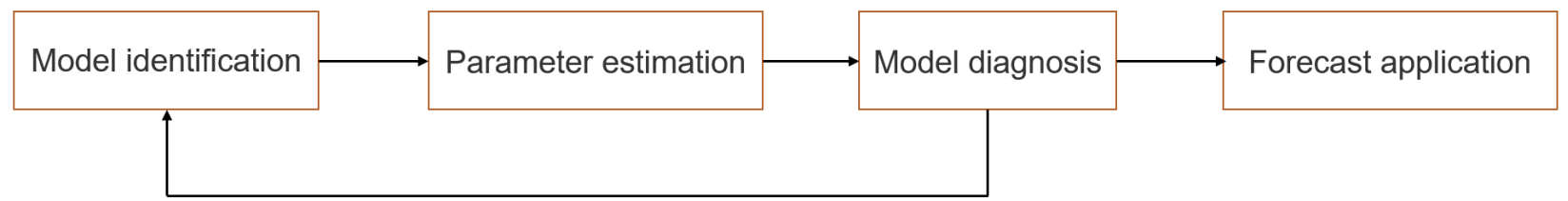

Figure 1.ARIMA modelling step diagram

\subsection{ARIMA model properties}

If $\left\{X_{t}\right\}$ obeys model $\operatorname{ARIMA}(\mathrm{p}, \mathrm{q}, \mathrm{d})$, and let $\varphi(B)=\Phi(B) \nabla^{d}$ and $\varphi(B)$ is called generalized autoregressive coefficient polynomials, then the stationarity of model $\operatorname{ARIMA}(p, q, d)$ depends entirely on the properties of the roots of $\varphi(B)=0$.

Since array $\left\{X_{t}\right\}$ is stable after difference of order $\mathrm{d}$, it can be set

$$
\Phi(B)=\prod_{i=1}^{p}\left(1-\lambda_{i} B\right),\left|\lambda_{i}\right|<1, i=1,2, \cdots, p
$$

thus,

$$
\varphi(B)=\Phi(B) \nabla^{d}=\left[\prod_{i=1}^{p}\left(1-\lambda_{i} B\right)\right](1-B)^{d}
$$

So, it's easy to know that $\operatorname{ARIMA}(p, q, d)$ has $p+d$ eigen roots, of which $p$ eigen roots are outside the unit circle, which are $\frac{1}{\lambda_{1}}, \cdots, \frac{1}{\lambda_{p}}$, and $\mathrm{d}$ of them are on the unit circle. Therefore, when $\mathrm{d} \neq 0$, the $\operatorname{ARIMA}(p, q, d)$ model is obviously non-stationary.

\subsection{ARIMA model building process}

\subsubsection{Stationary test}

Since the data is not stable, the stability test of the whole social electricity consumption data is carried out first. The simplest method to test the stationarity is to preliminarily judge the stationarity of time series through the broken line diagram or scatter diagram of time series. If the time series diagram shows that the image fluctuates randomly around a value and the range of fluctuation is bounded, it can be inferred that the time series is stationary by the nature of the stationary time series. If the sequence is non-stationary, then we have to do something to make it stationary. The most commonly used one is to carry out differential processing on the sequence, and then see whether the processed data has stationarity. If the first difference is still a non-stationary sequence, then the difference is carried out until it becomes a stationary sequence, and the number of difference is $\mathrm{d}$ in the model $\operatorname{ARIMA}(p, q, d)$.

Stabilization is an important basis for time series analysis. A time series $\{X t\}$ is strictly stationary if, for all times, the joint distribution of any positive integers $n$ and any $n$ positive integers $\left(t_{1}, \ldots t_{n}\right)\left(X_{t l}, \ldots X_{t k}\right)$ is the same as the joint distribution of $\left(X_{t 1+1}, \ldots X_{t n+1}\right)$ (in other words, 
strict stationarity requires that the joint distribution of $\left(X_{t}, \ldots X_{t n}\right)$ is invariant under the translation of time. This is a strong condition and difficult to test empirically. It is generally assumed that a weaker form of stationarity is assumed: if the mean of $X_{t}$ and the covariance of $X_{t}$ and $X_{t-l}$ are time-invariant, where $l$ is an arbitrary integer, then the time series $\left\{X_{t}\right\}$ is weakly stationary. More specifically, if

$$
\left\{\begin{array}{l}
E\left(X_{t}\right)=\mu, \\
\operatorname{Cov}\left(X_{t}, X_{t-l}\right)=\gamma_{l},
\end{array}\right.
$$

Where, $\mu$ is constant.

The time series $\{\mathrm{Xt}\}$ is weakly stationary. In practice, we can assume that $T$ values fluctuate by the same amount on either side of a constant horizontal line.

In the weakly stationary condition, there is actually an assumed condition implicit, that is, the first two order matrices of $X_{t}$ are finite.From this, we can think that if $X_{t}$ is strictly stationary and its first two moments are finite, then $X_{t}$ is also weakly stationary.On the contrary, it is generally not true, but if the time series $X_{t}$ is normally distributed, then the weak stationarity and the strict stationarity are equivalent.

The covariance $\operatorname{Cov}\left(X_{t}, X_{t-l}\right)=\gamma_{l}$ is called the autocovariance of the interval of phi.

Difference operation can stabilize a class of nonstationary time series. We can change the time series into a stationary series by the difference of order $d$.

A first order differential

$$
\nabla X_{t}=X_{t}-X_{t-1}-(1-B) X_{t}
$$

Where, $\nabla^{d}$ is called a difference operator of order $\mathrm{d}$.

$$
\begin{aligned}
& \nabla^{d}=(1-B)^{d}-1-\left(\begin{array}{l}
d \\
1
\end{array}\right) B+\left(\begin{array}{l}
d \\
2
\end{array}\right) B^{2}+\cdots+ \\
& (-1)^{d-1}\left(\begin{array}{l}
d \\
d-1
\end{array}\right) B^{d-1}+(-1)^{d} B^{d}
\end{aligned}
$$

To sum up, the time series model with the following structure is called summing autoregressive moving average model, namely ARIMA(p,q,d) model. The model is expressed as follows:

$$
\left\{\begin{array}{l}
\Phi(B) \nabla^{d} X_{t}=\Theta(B) \alpha_{t} \\
E\left(\alpha_{t}\right)=0, \operatorname{Var}\left(\alpha_{t}\right)=\sigma_{\alpha}^{2}, E\left(\alpha_{t}, \alpha_{s}\right)=0, s \neq t \\
E X_{s} \alpha_{t}=0, \forall s<t
\end{array}\right.
$$

on the type,

$$
\left\{\begin{array}{l}
\nabla^{d}=(1-B)^{d} \\
\Phi(B)=1-\Phi_{1} B-\cdots-\Phi_{p^{B}}{ }^{p} \\
\Theta(B)=1-\theta_{1} B-\cdots-\theta_{q} B^{q}
\end{array}\right.
$$

$\nabla^{d}=(1-B)^{d} \quad$ is the autoregressive coefficient polynomial of stationary $\operatorname{ARMA}(\mathrm{p}, \mathrm{q})$ model, $\Theta(B)=1-\theta_{1} B-\cdots-\theta_{q} B^{q}$ is the movement smoothing coefficient polynomial of stationary reversible $\operatorname{ARMA}(p, q)$ model.

All the above types, $\left\{\alpha_{t}\right\}$ are zero-mean white noise sequences.

In the $\operatorname{ARIMA}(\mathrm{P}, \mathrm{Q}, \mathrm{D})$ model,

When $d=0$, the $\operatorname{ARIMA}(p, q, d)$ model can be simplified to $\operatorname{ARMA}(p, q)$ model.

When $\mathrm{p}=0$, ARIMA $(\mathrm{p}, \mathrm{q}, \mathrm{d})$ model can be simplified to $\operatorname{IAM}(p, q)$ model.

When $q=0, \operatorname{ARIMA}(p, q, d)$ model can be simplified to $\operatorname{ARI}(p, q)$ model.

When $d=1$ and $p=q=0$, the $\operatorname{ARIMA}(p, q, d)$ model can be simplified as

$$
\left\{\begin{array}{l}
X_{t}=X_{t-1}+\alpha_{t} \\
E\left(\alpha_{t}\right)=0, \operatorname{Var}\left(\alpha_{t}\right)=\sigma_{\alpha}^{2}, E\left(\alpha_{t}, \alpha_{s}\right)=0, s \neq t \\
E X_{s} \alpha_{t}=0, \forall s<t
\end{array}\right.
$$

This is called the random walk model, or the drunk man model.

\subsubsection{Model recognition and hierarchy}

Model order $p, q$ should pass autocorrelation coefficient and partial autocorrelation coefficient diagram to identify, to the order of the model have a preliminary decision, a few primary $\mathrm{p}$ and $\mathrm{q}$ value combination model, the assessment of the effect of fitting of each model, select one of the best, using AIC criterion or BIC criterion to judge which model is the optimal,After calculating the AIC value and BIC value of each model, the smallest one is the optimal one.

AIC function :

$$
A I C=2 k+N \ln \frac{S S R}{N}
$$

BIC function :

$$
B I C=-2 \ln \left(\frac{S S R}{N}\right)+\ln (N) * k
$$

Where $\mathrm{k}$ is the number of parameters, $\mathrm{N}$ is the number of observers, and SSR (Sum SQAURE OF RESIDUE) is the sum of squares.

\subsubsection{Model parameter estimation and verification}

Model significance test is to see whether the model fits the time series well, that is, whether a model is significantly effective.The criterion to measure the effectiveness is whether the model extracts the sequence value information sufficiently.A significantly effective model should extract most of the information in the sequence, that is, there is no longer any available information in the residual term fitted by the model, that is, the residual sequence should be a white noise sequence.Therefore, whether the model is significant depends on whether the residual sequence is white noise.If the residual sequence is white noise sequence, the model is considered to be reasonable and can be predicted; otherwise, it means that part of the residual information in the time series data has not been extracted, and the model needs to be further improved and other 
models are selected for re-fitting.Therefore, the significance test of the model is to check whether the residual sequence of the model is white noise sequence.

The significance test of parameters refers to the test of whether the significance of parameters in the model is zero. The purpose of the test is to make the model more concise. If a parameter in the model is not significant, it means that the variable corresponding to the parameter has no obvious influence on the overall model, so this variable should be deleted from the fitting model, and the final model is composed of a series of non-zero variables.

Hypotheses,

$$
H_{0}: \beta_{j}=0 \leftrightarrow H_{1}: \beta_{j} \neq 0
$$

Test statistics,

$$
T=\sqrt{T-m} \frac{\widetilde{\beta_{j}}-\beta_{j}}{\sqrt{a_{j j} Q(\widetilde{\beta})}} \sim t(T-m)
$$

$H_{0}$ residual-difference sequence is classified as white noise sequence and $H_{1}$ is non-white noise sequence, and the maximum likelihood estimation $\widehat{\beta}_{j}$ is asymptotically unbiased estimation of parameters $\beta_{j}$, which has asymptotic normality. $a_{j j} Q(\widetilde{\beta})$ is denoted $V\left(\beta_{j}\right)$ by the $j^{*} j$ element, then the $\widehat{\beta}_{j}$ asymptotic distribution is $N\left(\beta_{j}, T^{-1} a_{j j} Q(\widetilde{\beta})\right)$, and the test level is $\alpha$, so the rejection domain of detection can be obtained as:

$$
\left\{|t| \geq t_{1-\frac{\alpha}{2}}(T-m)\right\}
$$

\subsubsection{Model prediction}

After passing the above tests, it is shown that this model has a good degree of fluctuation in fitting time series values, but this model is not unique. We try to use the model to predict the result, and then compare it with the real value to observe the value of relative error, and take the model with small relative error as the final model. Therefore, the final model is used to predict the future value of time series, and it is applied to practical problems.

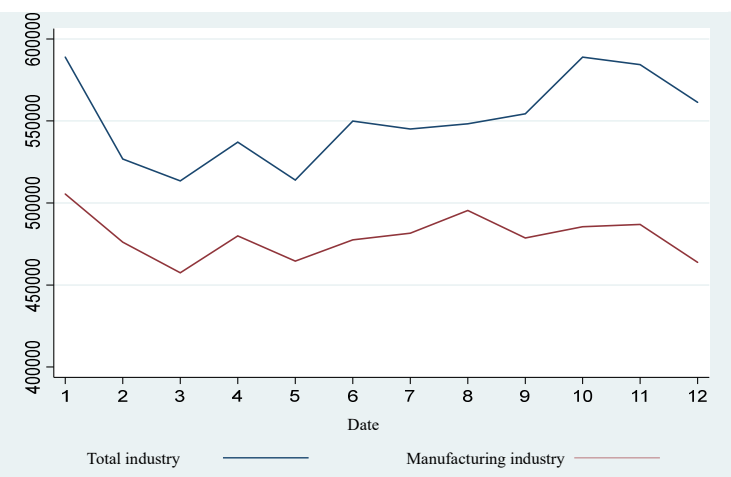

\section{Analysis results and discussion}

According to the characteristics of industrial structure in Western China, the energy consumption structure is divided into six types: agriculture, forestry, animal husbandry and fishery, industry, construction, transportation, postal storage, wholesale, retail, catering and accommodation, and urban and rural residents' life. Energy consumption is represented by four statistical indicators of total energy consumption by industry, namely total energy consumption, raw coal consumption, oil and gas consumption and electricity consumption.

According to different fields such as agriculture, forestry, animal husbandry and fishery, industry, construction, transportation, postal storage, wholesale, retail, catering and accommodation, and urban and rural residents' life, the analysis results of energy structure are shown in Figure 2.

To sum up, manufacturing power consumption accounts for more than $80 \%$ of the total industrial power consumption, and manufacturing power consumption plays a decisive role in the total industrial power consumption; Among the total power consumption of agriculture, forestry, animal husbandry and their auxiliary activities, the power consumption of agriculture, forestry, animal husbandry and fishery accounts for more than $90 \%$, among which the power consumption of auxiliary activities of agriculture, forestry, animal husbandry and fishery has a decisive impact on the total power consumption of Agriculture, forestry, animal husbandry and fishery; In the total power consumption of construction industry, the power consumption of housing construction, building decoration and other construction industries accounts for $73 \%-83 \%$. The power consumption of building decoration and other construction industries mainly affects the change trend of the total power consumption of construction industry; In the total power consumption of transportation, postal and storage industry, the power consumption of railway transportation and road transportation accounts for more than $91 \%$, and the power consumption of railway transportation accounts for $76 \%-83 \%$, which is decisive to the total power consumption; In the total power consumption of urban and rural residents, the total power consumption of urban residents accounts for $66 \%-72 \%$, while the total power consumption of rural residents only accounts for $28 \%$ $34 \%$. The gap is huge, and there is a trend of first rising and then falling.

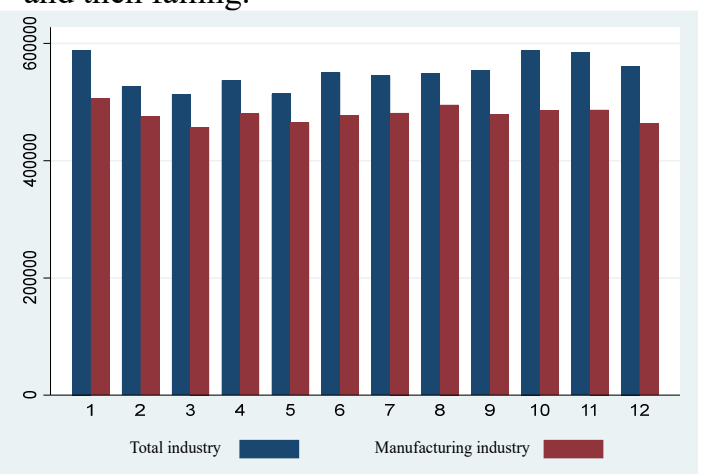

(a) Industrial field 

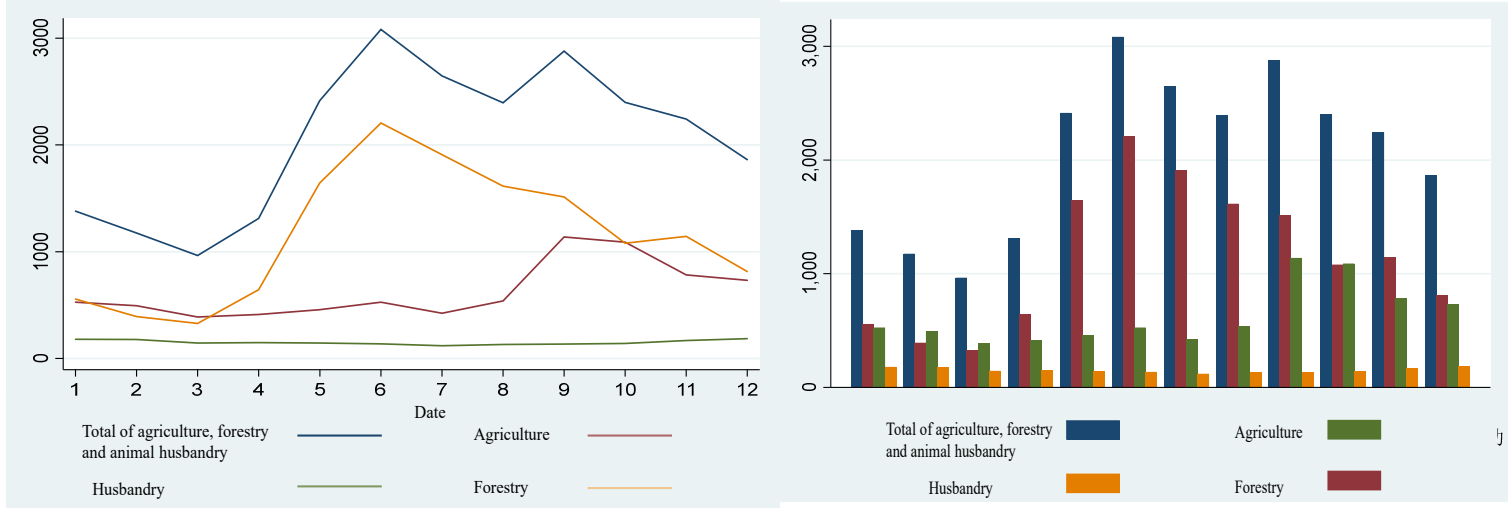

(b) Agriculture, forestry, animal husbandry and fishery
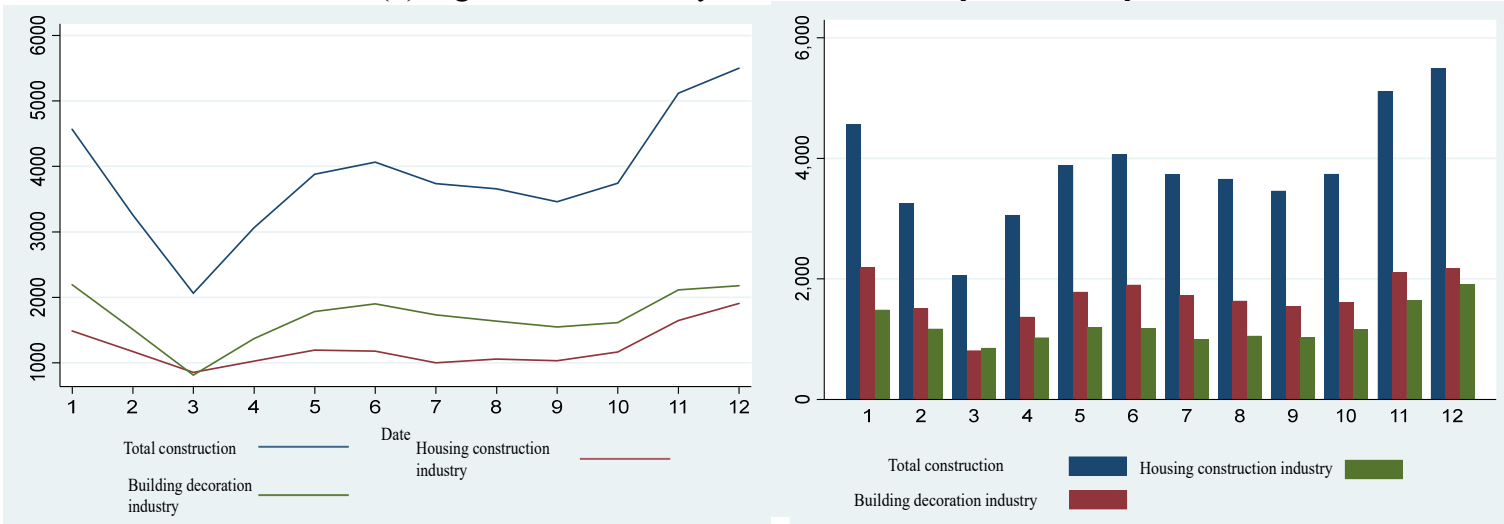

(c) Construction industry
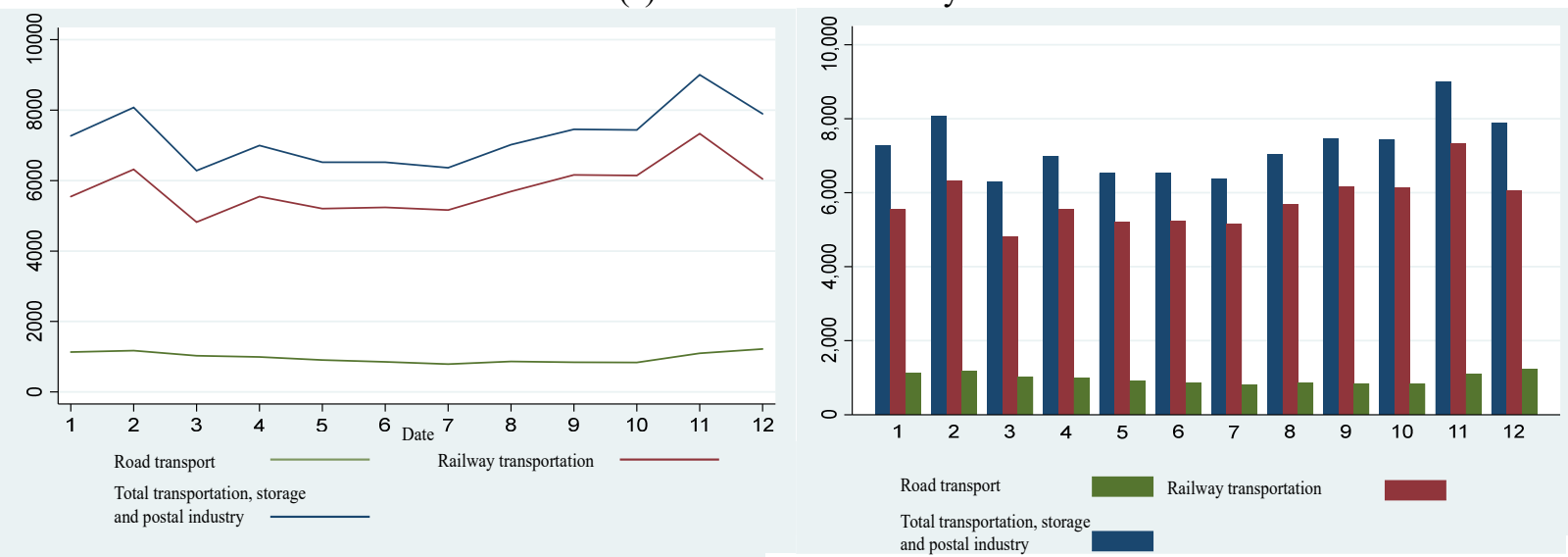

(d) Transportation, post and storage
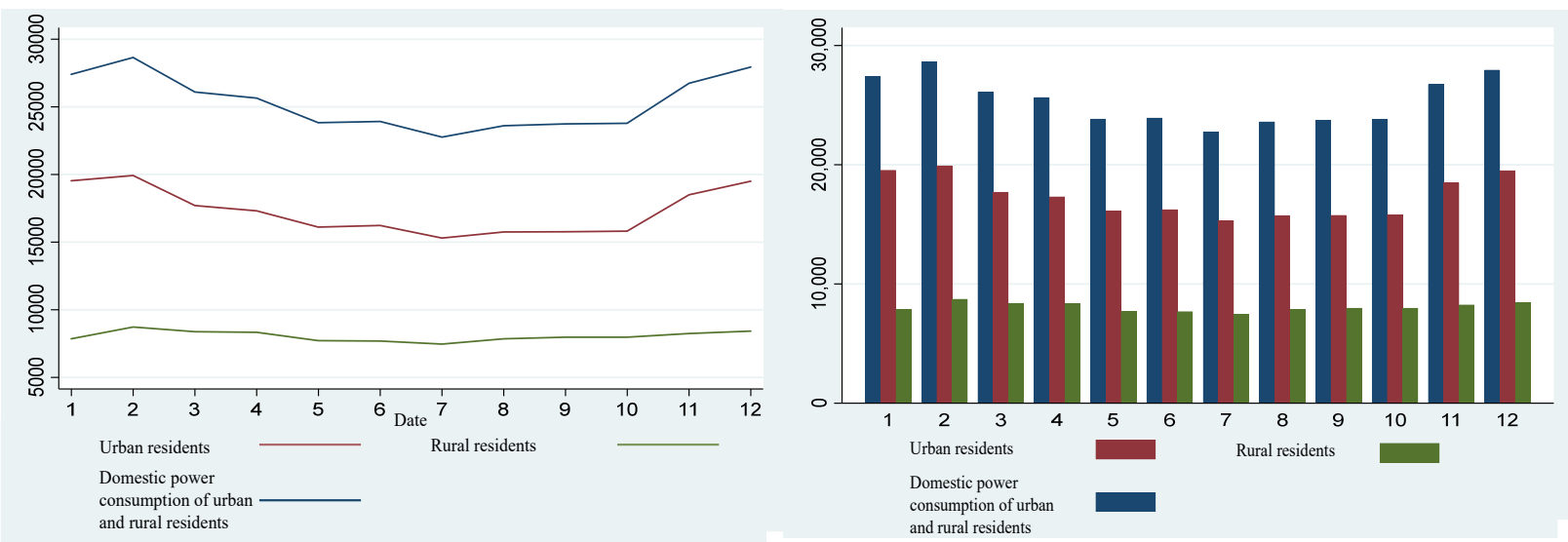

(e) Life field of urban and rural residents

Figure 2. Analysis results of energy consumption structure in different fields 


\section{Conclusion}

(1) Through technology promotion to improve the power consumption efficiency of end user departments, and expand the promotion of energy-saving technology to reduce energy consumption

The power consumption of manufacturing industry in Western China accounts for a large proportion of the total industrial power consumption. How to reduce the power consumption of manufacturing industry has become the main factor to reduce the total industrial power consumption. In the manufacturing industry, the chemical raw materials and chemical products manufacturing industry, ferrous metal smelting and rolling processing industry, and non-ferrous metal smelting and rolling processing industry consume a large amount of energy, so the power consumption efficiency can be improved through technological innovation, The development and application of energy saving equipment is the key to energy saving in manufacturing industry. In the transportation, postal and storage industry, it is difficult to reduce the power consumption of railway transportation, but the total power consumption of road transportation can be reduced, for example, the energy consumption per unit distance of electric vehicles can be reduced by technical means.

(2) The government strengthens the supervision mechanism to the electric power energy utilization

In the total power consumption of urban and rural residents, we can see that the power consumption of urban residents is 3-4 times that of rural residents. Therefore, the government needs to strengthen the supervision of urban residents to make them use electricity reasonably and effectively, which is of great significance to reduce the total power consumption of urban and rural residents. From the figure, we can see that the power consumption of rural residents remains almost unchanged in the four seasons, but urban residents fluctuate. The main reason is that the power consumption in winter shows an upward trend. Maybe it can be rectified by the way of general central heating.

(3) Accelerating the intellectualization of power consumption Encourage the construction of smart home, smart building, smart community and smart factory with smart terminal and flexible power trading as the main features to support the construction of smart city. Strengthen DSM, popularize intelligent energy monitoring and diagnosis technology, accelerate the construction of energy management center of industrial enterprises, and build information service platform based on Internet. Build a micro balance system characterized by multi energy integration, open sharing, two-way communication and intelligent control, and flexible integration of various energy consumption terminals. Build access facilities and information service platform for energy consumers at different levels of families, parks and regions to participate in the energy market.

\section{ACKNOWLEDGMENTS}

This research was financially supported by the SGCC Technology Project- The research on integrated simulation method and practical technology of think tank research platform.

\section{Reference}

1. YANG Yang, ZHANG Qian-qian, Low Carbon Economy Development Paths in Beijing-TianjinHebei Region under Carbon Reduction Target of Absolute Constraints[J]. Soft Science, 29,11 (2015):105-109.

2. Martins, John, The impact of the use of energy sources on the quality of life of poor communities[J]. Social Indicators Research,72(2005):373-402.

3. Leopold, Armin, Energy related system dynamic models: a literature review[J].Central European Journal of Operations Research,24,1(2016):231-261.

4. Zhang Zongyi, Guo Xiaoming, Wang Feng, Energy Price Impacts on Energy Efficiency of the Tertiary Industry in China--A VAR-model Based Empirical Study[J]. MANAGEMENT REVIEW, 22,6(2010): 61-70.

5. TANG Baojun, ZHOU Huiling, CAO Hong, WEI Yiming, Carbon Emissions and Economic Growth in APEC Members with Implications for China[J]. Chinese Journal of Management, 12,12(2015):18651871.

6. CUI Baisheng, ZHU Lin, Research on Economic Growth and Carbon Abatement Under the Energy Consumption Controlling Objective Based on Endogenous Growth Theory and GVAR Model[J]. Chinese Journal of Management Science, 24,1(2016):11-20.

7. LIU Qia,ZHAO Qiu-hong, Policy impacts on power generation enterprises' decisions and optimization model[J].Systems Engineering - Theory \& Practice,35,7(2015):1717-1725. 